\title{
Expression profiles of podocytes exposed to high glucose reveal new insights into early diabetic glomerulopathy
}

\author{
Sanjay Jain ${ }^{1,2}$, Laura De Petris ${ }^{3}$, Masato Hoshi ${ }^{1}$, Shreeram Akilesh², Rajshekhar Chatterjee ${ }^{1}$ and Helen Liapis ${ }^{1,2}$
}

Podocyte injury has been suggested to have a pivotal role in the pathogenesis of diabetic glomerulopathy. To glean insights into molecular mechanisms underlying diabetic podocyte injury, we generated temporal global gene transcript profiles of podocytes exposed to high glucose for a time interval of 1 or 2 weeks using microarrays. A number of genes were altered at both 1 and 2 weeks of glucose exposure compared with controls grown under normal glucose. These included extracellular matrix modulators, cell cycle regulators, extracellular transduction signals and membrane transport proteins. Novel genes that were altered at both 1 and 2 weeks of high-glucose exposure included neutrophil gelatinaseassociated lipocalin (LCN2 or NGAL, decreased by 3.2-fold at 1 week and by 7.2-fold at 2 weeks), endothelial lipase ( $E L$, increased by 3.6 -fold at 1 week and 3.9-fold at 2 week) and UDP-glucuronosyltransferase 8 (UGT8, increased by 3.9-fold at 1 week and 5.0-fold at 2 weeks). To further validate these results, we used real-time PCR from independent podocyte cultures, immunohistochemistry in renal biopsies and immunoblotting on urine specimens from diabetic patients. A more detailed time course revealed changes in LCN2 and EL mRNA levels as early as 6 hours and in UGT8 mRNA level at 12 hours post high-glucose exposure. EL immunohistochemistry on human tissues showed markedly increased expression in glomeruli, and immunoblotting readily detected EL in a subset of urine samples from diabetic nephropathy patients. In addition to previously implicated roles of these genes in ischemic or oxidative stress, our results further support their importance in hyperglycemic podocyte stress and possibly diabetic glomerulopathy pathogenesis and diagnosis in humans.

Laboratory Investigation (2011) 91, 488-498; doi:10.1038/labinvest.2010.188; published online 22 November 2010

KEYWORDS: diabetic glomerulopathy; expression profiles; nephropathy; podocytes

Diabetic nephropathy, a clinical syndrome of persistent microalbuminuria and a common complication of diabetes, is currently the leading cause of end-stage renal disease (ESRD) in the United States. Albuminuria indicates excessive albumin filtration from the glomerulus into the tubules, which overwhelms their metabolic capacity promoting local inflammation and tubulointerstitial scarring known as diabetic nephropathy. A central initial event in the albuminuriaESRD sequence is the podocyte injury. ${ }^{1-3}$ The podocyte consists of a cell body, primary and secondary foot processes, and the slit diaphragm (filtration barrier). It is proposed that hyperglycemia causes podocyte oxidative stress-defined as the damage caused by reactive oxygen species-which leads to foot process effacement, followed by podocyte apoptosis. ${ }^{4,5}$ For example, in the Akita model of type 1 diabetes, or the leptin receptor-deficient $\mathrm{db} / \mathrm{db}$ mouse model of type 2 DKD, podocytes lose nephrin expression (the major component of the slit diaphragm), foot processes become effaced and eventually detach from the glomerular basement membrane, undergoing death by apoptosis. ${ }^{4,5}$ Podocyte apoptosis is in part mediated by transforming growth factor- $\beta$ (TGF $\beta$ ) signaling and possibly epithelial-mesenchymal transformation. ${ }^{6-8}$ For example, Li et $a^{8}{ }^{8}$ show that TGF $\beta$ under conditions of high glucose (HG) suppresses expression of key slit diaphragm proteins, induces extracellular matrix protein expression (eg, fibronectin and collagen I) and leads to

\footnotetext{
${ }^{1}$ Renal Division, Department of Internal Medicine, Washington University, School of Medicine, St Louis, MO, USA; ${ }^{2}$ Department of Pathology \& Immunology, Washington University, School of Medicine, St Louis, MO, USA and ${ }^{3}$ Department of Pediatrics, San Benedetto del Tronto Hospital, San Benedetto del Tronto, Italy Correspondence: Dr Sanjay Jain, MD, PhD, Renal Division, Departments of Medicine, Pathology and Immunology, 660 S. Euclid Avenue, Box 8126, St Louis, MO 63110, USA and Dr Helen Liapis, MD, Department of Pathology \& Immunology, Washington University School of Medicine, 660 S. Euclid Avenue, Campus Box 8118 , St Louis, MO 63110-1093, USA.
}

E-mails: SJain22@WUSTL.EDU and Liapis@path.wustl.edu

Received 26 April 2010; revised 17 September 2010; accepted 12 October 2010 
secretion of matrix metalloproteinase-9 (MMP-9). However, activation of other metabolic pathways, eg, the polyol pathway, ${ }^{9}$ protein kinase $\mathrm{C},{ }^{10}$ the hexoamine pathway, ${ }^{11-14}$ also has a role in diabetic nephropathy. Recently, VEGF-mediated signaling has also been implicated in this process, ${ }^{15,16}$ and hyperglycemia mimicking hypoxic injury to endothelial cells is proposed. ${ }^{17,18}$ These studies demonstrate that the molecular pathogenesis of diabetic podocyte injury is likely multifactorial involving a number of interrelated signaling pathways that have yet to be well understood. Understanding the molecular milieu of diabetic podocyte injury, an early event in diabetic nephropathy, remains a primary target in identifying novel avenues for early intervention and prevention of severe late complications of this increasingly prevalent disease.

We have previously found that BMP7 confers podocyte resistance to hyperglycemic injury by restoring major podocyte proteins such as synaptopodin and podocin. ${ }^{19}$ Identifying new molecular changes and integrating them to known pathways are important in obtaining deeper insights into the mechanism of diabetic nephropathy, early diagnosis and possible new therapies. One approach towards this end is using high-throughput methods that detect multiple molecular changes simultaneously. For example, microarray technology enables one to measure gene expression in whole genomes to identify new genes and pathways associated with a disease. Microarray studies on whole diabetic kidney and/or on mesangial cells have found altered gene expression in the early phases of diabetic injury in mice. ${ }^{20-24}$ Although these studies provide initial insights into early global changes in the diabetic kidney, little is known about the molecular and temporal events occurring specifically in podocytes. Recently, early global changes due to HG were reported in podocytes. ${ }^{25}$ Information regarding molecular changes in podocytes due to prolonged high-glucose exposure will help derive important insights into how these may lead to diabetic nephropathy, a disease that develops over a long time. This will also enhance the possibility of discovering biomarkers that can help discern podocyte dysfunction over broad range of this disease.

The aim of this study was to examine the effect of prolonged high-glucose exposure on mRNA expression profiles in mouse podocytes. Using expression microarrays, we discovered genes that are strongly and consistently associated with hyperglycemic podocyte stress in a time-course analysis. The in vitro podocyte injury alterations were also detected in renal biopsies and urine samples from patients with diabetic nephropathy, suggesting that these may be relevant to the pathogenesis of diabetic glomerular disease in humans.

\section{MATERIALS AND METHODS Cell Culture}

Experiments were performed using a thermosensitive SV-40transfected immortalized mouse podocyte cell line (gift from Peter Mundel, Mount Sinai School of Medicine, New York).
Podocytes were cultured in RPMI 1640 supplemented with $10 \%$ fetal bovine serum, $100 \mathrm{U} / \mathrm{ml}$ penicillin, and $100 \mu \mathrm{g} / \mathrm{ml}$ streptomycin. Cells were grown at $33^{\circ} \mathrm{C}$ and were treated with $10 \mathrm{U} / \mathrm{ml}$ of mouse recombinant $\gamma$-interferon (Sigma, St Louis, MO, USA), as previously described. ${ }^{19}$ At confluence, podocytes were maintained on a bed of type I collagen at $37^{\circ} \mathrm{C}$ for 14 days without $\gamma$-interferon to allow differentiation. ${ }^{26}$ These conditionally immortalized cells were then either exposed to media containing normal glucose (NG) as a control (5.5 mmol/l D-glucose) or HG (25 mmol/l D-glucose) for $6,12,18,24$ and $72 \mathrm{~h}$, and 1 or 2 weeks. Experiments were performed in duplicate.

To account for the osmotic effect of HG, podocytes were starved for 24 hours with media containing mannitol. In this model, 1-week exposure to HG was considered an arbitrary representation of sub-acute HG stress, and an arbitrary representation of sustained stress was taken as HG exposure for 2 weeks. Exposure of podocytes to HG from 6-72 hours was performed to study temporal alterations and to independently validate the microarray results.

\section{RNA Extraction}

Total RNA was extracted with TRIzol reagent according to manufacturer's protocol (Invitrogen Inc.). An additional cleanup step was used by employing an RNeasy Mini Kit (QIAGEN Inc., Valencia, CA, USA). RNA concentration and purity was assessed by spectroscopy (Nanodrop) with the A260/A280 ratio in the range $1.77-2.08$. RNA samples were used immediately or stored at $-80^{\circ} \mathrm{C}$ for subsequent microarray analysis or for real-time PCR.

\section{Microarray Hybridization and Data Analysis}

Total RNAs $(1-5 \mu \mathrm{g})$ extracted from podocytes cultured in HG for 1 or 2 weeks and in NG for 2 weeks (in duplicate) were reverse transcribed into cDNA, and biotin-labeled cRNA targets were generated. The cRNA was fragmented and hybridized to Affymetrix GeneChip Mouse Genome 4302.0 microarrays (Affymetrix, Santa Clara, CA, USA) at the Laboratory of Translational Pathology Microarray Core Facility of Washington University, as previously described. ${ }^{27}$ Quantitative analysis of hybridization patterns and intensities was performed by Affymetrix software, and the resulting data were analyzed by Affymetrix Microarray Suite software (Version 5.0). The entire raw data are available at http:// bioinformatics.wustl.edu. The data were scaled to 1500 units of signal intensity for comparisons across samples and imported into dCHIP for analysis. The data were filtered to exclude genes that were not expressed or did not vary, using the coefficient of variation (s.d./mean) and percentage of presence calls in the arrays. First, the genes were filtered such that the coefficient of variation across samples was $>0.5$ and $<1000 .^{28,29}$ Second, only the genes that had a presence call percentage of $\geq 20 \%$ across the arrays were included for analysis. After filtering, a list of 1790 genes was used to identify differentially expressed genes in the data set. Using 
the replicate NG arrays as the baseline (B), the replicate $1 \mathrm{~W}$ or $2 \mathrm{~W}$ array experiments $(\mathrm{E})$ were each compared for changes in gene expression using a threefold cutoff $(\mathrm{B} / \mathrm{E}$ or $\mathrm{E} / \mathrm{B}>3)$, absolute signal difference $>100(\mathrm{~B}-\mathrm{E}$ or $\mathrm{E}-\mathrm{B}>100)$ and a presence call percentage of $\geq 20 \%$ in each of the baseline and experimental data sets. The raw signal intensity data for the resulting differentially expressed genes were reviewed, and only those genes that showed close agreement between the duplicates were pursued.

\section{Real-Time PCR}

Total RNA extracted from podocytes cultured (duplicates) in NG or HG in various time intervals (6 hours to 2 weeks) was converted into cDNA and amplified by real-time PCR in 'one-step' reaction (Qiagen, OneStep RT-PCR, Germantown, MD, USA). The SYBR Green was used as fluorogenic probe system. PCR kinetics and data quantification were performed with 4000 Multiplex Quantitative PCR System Software (Stratagene, La Jolla, CA, USA). Quantification of the target gene was performed according to the standard curve method. ${ }^{30}$ mRNA levels were normalized to $\beta$-actin. Experiments were performed in triplicate. We used the following primers: UDP-glucuronosyltransferase 8 (Ugt8) 5'-CCCACTGCCAGA AGATCTGC-3'; $3^{\prime}$-TGGAATAGCAAGGGCTGCTAA-5'; endothelial lipase (EL) 5'-GAGCGAGCCGTACACCTCTT-3', $3^{\prime}$-TGGATACGCTGGCAACTTTG-5'; LCN 5'-GATGCGCAG AGACCCAATG- ${ }^{\prime}$, $3^{\prime}$-AGGAACGTTTCACCCGCTTT- ${ }^{\prime}$.

\section{Human Tissue Studies}

Paraffin-embedded tissue blocks or fresh cryopreserved tissue from renal biopsies from patients $(n=8)$ with diabetic glomerulosclerosis were randomly retrieved from the files of the Department of Pathology and Immunology at Washington University in St Louis or George M. O'Brien Center for Kidney Disease Research Kidney Translational Research Core at Washington University. All specimens used for the research were collected under protocols approved by the Institutional Review Board of Washington University School of Medicine. Light microscopy, routine immunofluorescence and electron microscopy were retrospectively reviewed to ascertain diagnosis. Seven controls consisted of histologically normal nondiabetic kidneys.

\section{Immunohistochemistry}

Immunohistochemistry on formalin-fixed paraffin-embedded sections $(4 \mu \mathrm{m})$ was performed using antigen retrieval for 15 minutes $(10 \mathrm{mM}$ sodium citrate, $0.05 \%$ Tween20, pH 6.0) and peroxidase method. HematoxylinEosin was used for counterstaining. For cryopreserved tissue, immunofluorescence was performed on $10-\mu \mathrm{m}$ sections that were post-fixed in PBS containing 4\% paraformaldehyde, washed and blocked with Image-iT ${ }^{\mathrm{TM}}$ FX signal enhancer (Invitrogen Inc.) for 30 minutes and were then incubated with the primary antibody. The primary and secondary antibodies used were anti-EL (1:50, Cayman chemical company
Cat\# 100030) and biotinylated anti-rabbit (1:200, Jackson Immunoresearch Inc.), respectively. WT1 antibody (1:10, Santa Cruz Biotechnology Inc.) was used to label podocytes in biopsies. The signals were visualized using streptavidinHRP (1:400, JacksonImmunoresearch Inc.) for paraffin embedded tissue, streptavidin-alexa594 (molecular probes) for EL immunofluorescence and strepatavidin-alexa488 for WT1 immunofluorescence. Slides were incubated with bis-benzamide (Sigma) for 5 minutes to visualize nuclei. Nikon $80 \mathrm{i}$ upright microscope (Nikon) equipped with CoolSnapES camera (photometrics) was used to capture the images, and Nikon Elements (Nikon) and Adobe Photoshop (Adobe) softwares were used for image processing.

\section{Immunoblotting}

SDS-PAGE (10\%) was performed on urine specimens $(15 \mu \mathrm{l}$ each) of four patients with diabetic nephropathy and three individuals with no known kidney disease (controls) using standard procedures. The proteins were transferred to PVDF membrane using Bio-Rad semi-dry apparatus. After washing twice with TBS buffer, the PVDF membrane was blocked using $2 \%$ non-fat milk and $2 \%$ BSA in TBST buffer for 1 hour at room temperature, and then incubated with rabbit anti-EL (1:100) and mouse anti-albumin (1:2000, Invitrogen Cat\#03-0700) antibodies for 16 hours at $4{ }^{\circ} \mathrm{C}$. After washing with TBST, the membranes were incubated for 1 hour at $25^{\circ} \mathrm{C}$ with IRDye800CW-conjugated goat anti-rabbit IgG (1:2000, red) and IRDye680-conjugated goat anti-mouse IgG (1:2000, green) secondary antibodies (LI-COR Biosciences). After washing, the respective antigens were visualized on an Odyssey Infrared Imaging System (LI-COR Biosciences) with both 700- and 800-nm channels. For confirming the specificity of EL antibody, different amounts of purified human Albumin (Sigma) ranging from $0.1-50 \mu$ g were blotted on two separate nitrocellulose membranes, and immunoblotting and detection was performed as described above for albumin and EL.

\section{Statistical Analysis}

In real-time PCR experiments, a two-way analysis of variance was used to compare control with experimental groups. Results are shown as the mean \pm s.d. $P<0.05$ was considered statistically significant.

\section{RESULTS}

To identify podocyte-specific genes potentially regulated by HG exposure, we treated podocytes in culture for 1 or 2 weeks with HG and compared their expression profiles with controls grown in NG for 2 weeks using Affymetrix Mouse Genome 4302.0 microarrays. A filtered set of 1790 genes was examined for changes in gene expression. Compared with NG, there were 19 transcripts that were significantly downregulated at 1 week of $\mathrm{HG}$ exposure (Tables 1 and 2, see Materials and methods). These include: brain expressed gene 1 (Bex1), thyroid hormone receptor interactor 11 (Trip11) and 
Table 1 Genes downregulated and upregulated in podocytes treated with high glucose for 1 week compared with podocytes cultured in normal glucose conditions

\begin{tabular}{|c|c|c|c|}
\hline Probe set & Downregulated gene & Accession & Fold change \\
\hline 1448595_a_at & Bex1: brain-expressed gene 1 & NM_009052 & -6.61 \\
\hline 1443153_at & Trip11: thyroid hormone receptor interactor 11 & BB306866 & -4.21 \\
\hline 1458269_at & Pcdh9: protocadherin 9 & AW048370 & -3.97 \\
\hline 1427747_a_at & Len2: lipocalin 2 & $\mathrm{X} 14607$ & -3.82 \\
\hline 1442704_at & Mm.214935.1 & BM250739 & -3.58 \\
\hline 1450154_at & Folh1: folate hydrolase & NM_016770 & -3.54 \\
\hline 1445426_at & Mm.42287.1 & BB457090 & -3.36 \\
\hline 1444229_at & Nr2f2: nuclear receptor subfamily 2 , group $F$, member 2 & BB053811 & -3.34 \\
\hline 1456659_at & LOC552902: hypothetical LOC552902 & BM116906 & -3.2 \\
\hline 1424375_s_at & Gimap4: GTPase, IMAP family member 4 & BC005577 & -3.19 \\
\hline 1439224_at & Mm.133637.1 & BB373816 & -3.17 \\
\hline 1443145_at & $\begin{array}{l}\text { Apbb1ip: amyloid beta (A4) precursor protein-binding, family } B \text {, } \\
\text { member } 1 \text { interacting protein }\end{array}$ & BB153348 & -3.12 \\
\hline 1454589_at & 9430006E15Rik: RIKEN cDNA 9430006E15 gene & AK020405 & -3.08 \\
\hline 1459750_s_at & Gpr123: G protein-coupled receptor 123 & AU015577 & -3.01 \\
\hline Probe set & Upregulated gene & Accession & Fold change \\
\hline 1435330_at & Pyhin 1: pyrin and HIN domain family, member 1 & BM241008 & 4.28 \\
\hline 1437937_at & Ccbp2: chemokine binding protein 2 & AV220666 & 4.13 \\
\hline 1442844_at & A830052D11Rik: RIKEN cDNA A830052D11 gene & BB271008 & 3.86 \\
\hline 1431315_at & Hyls1: hydrolethalus syndrome 1 & BM570636 & 3.85 \\
\hline 1447870_x_at & 1110002E22Rik: RIKEN cDNA 1110002 E22 gene & BB099116 & 3.81 \\
\hline 1418676_at & Is/2: insulin related protein 2 (islet 2) & NM_027397 & 3.79 \\
\hline 1444199_at & Mm.45087.1 & AW046689 & 3.69 \\
\hline 1425145_at & \|11r/1: interleukin 1 receptor-like 1 & D13695 & 3.58 \\
\hline 1422691_at & Spt/c1: serine palmitoyltransferase, long-chain base subunit 1 & AF003823 & 3.36 \\
\hline 1421262_at & Lipg: lipase, endothelial & BC020991 & 3.22 \\
\hline 1419063_at & Ugt8a: UDP galactosyltransferase $8 \mathrm{~A}$ & NM_011674 & 3.14 \\
\hline 1449356_at & Asb5: ankyrin repeat and SOCs box-containing 5 & NM_029569 & 3.06 \\
\hline 1449473_s_at & Cd40: CD40 antigen & NM_011611 & 3.04 \\
\hline 1439043_at & Tra2a: transformer 2 alpha homolog (Drosophila) & BE982794 & 3.01 \\
\hline
\end{tabular}

A cut off of threefold changes was used to filter the data (see Materials and Methods). Genes in bold were used for further studies, see Table 3 and Figures $1,2$. 
Table 2 Genes downregulated and upregulated in podocytes treated with high glucose for two week compared with podocytes cultured in normal glucose conditions

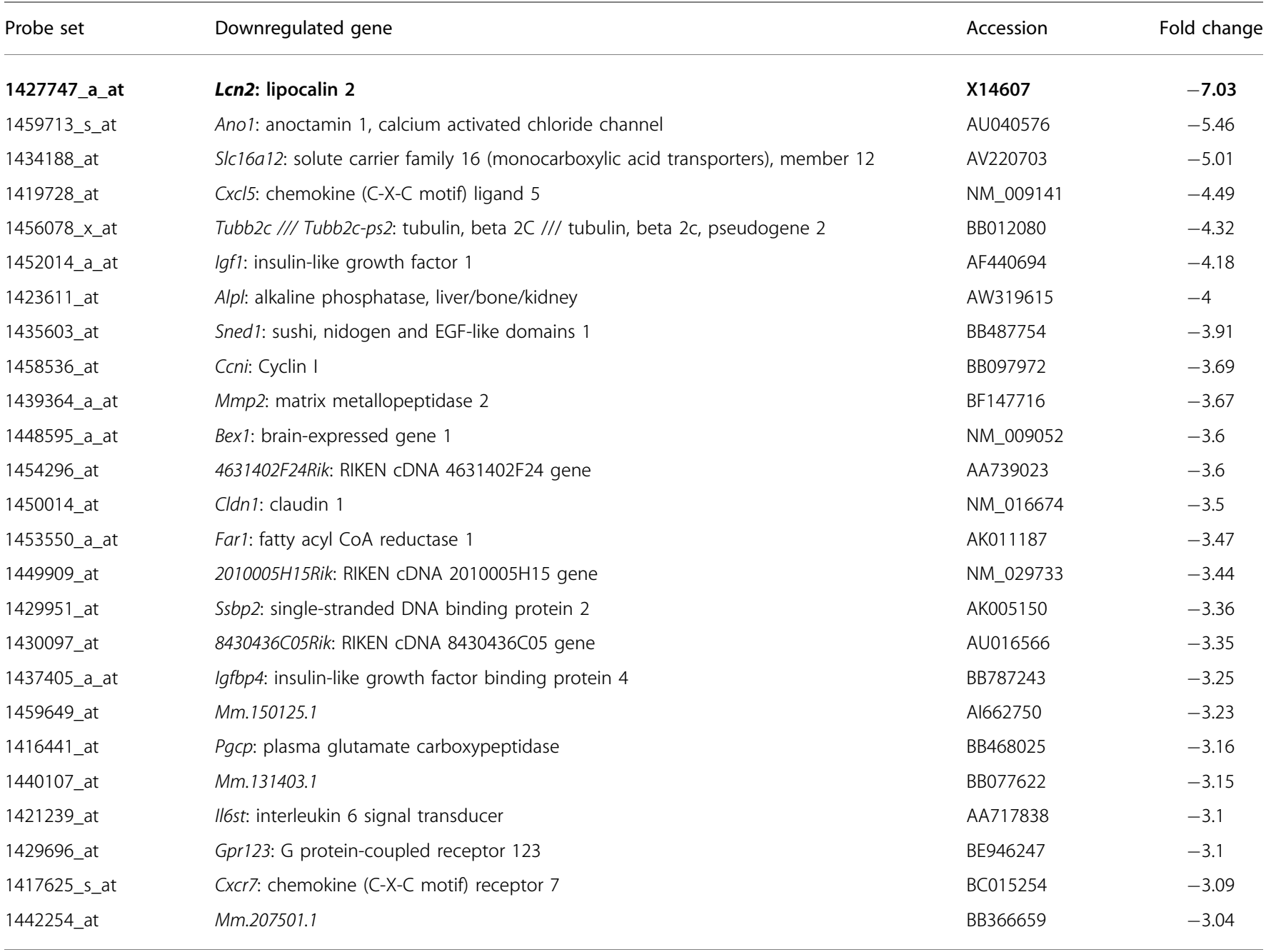

Probe set

\section{Probe set}

\section{0_at}

1419063_at

1444199_at

1431315_at

1449751_at

1422944_a_at

1430786_at

1426278_at

1427184_at

1421262_at

1422155_at

1455730_at

1441757_at

1430419_at

1421350_a_at
Upregulated gene

Mm.28870.2

Ugt8a: UDP galactosyltransferase $8 \mathrm{~A}$

Mm.45087.1

Hyls 1: hydrolethalus syndrome 1

Slc6a6: solute carrier family 6 (neurotransmitter transporter, taurine), member 6

Diap3: diaphanous homolog 3 (Drosophila)

1110002E22Rik: RIKEN cDNA 1110002 E22 gene

Ifi2712a: interferon, alpha-inducible protein 27 like 2A

Tcrb-J: T-cell receptor beta, joining region

\section{Lipg: lipase, endothelial}

Hist2h3c2: histone cluster 2, $\mathrm{H} 3 \mathrm{c} 2$

Dlgap5: discs, large (Drosophila) homolog-associated protein 5

1190002F15Rik: RIKEN cDNA $1190002 F 15$ gene

2310031A07Rik: RIKEN CDNA 2310031A07 gene

Grip1: glutamate receptor interacting protein 1
Accession

Fold change

B1651113

13.95

NM_011674

5.26

AW046689

4.89

BM570636

4.59

AA589629

4.57

NM_019670

4.46

BE991102

4.39

AY090098

4.37

BF318536

3.97

BC020991

3.95

$\begin{array}{ll}\text { BC015270 } & 3.79\end{array}$

BM250919 $\quad 3.67$

Al120476 $\quad 3.65$

AK009549 $\quad 3.64$

NM_130891 3.58 
Table 2 Continued

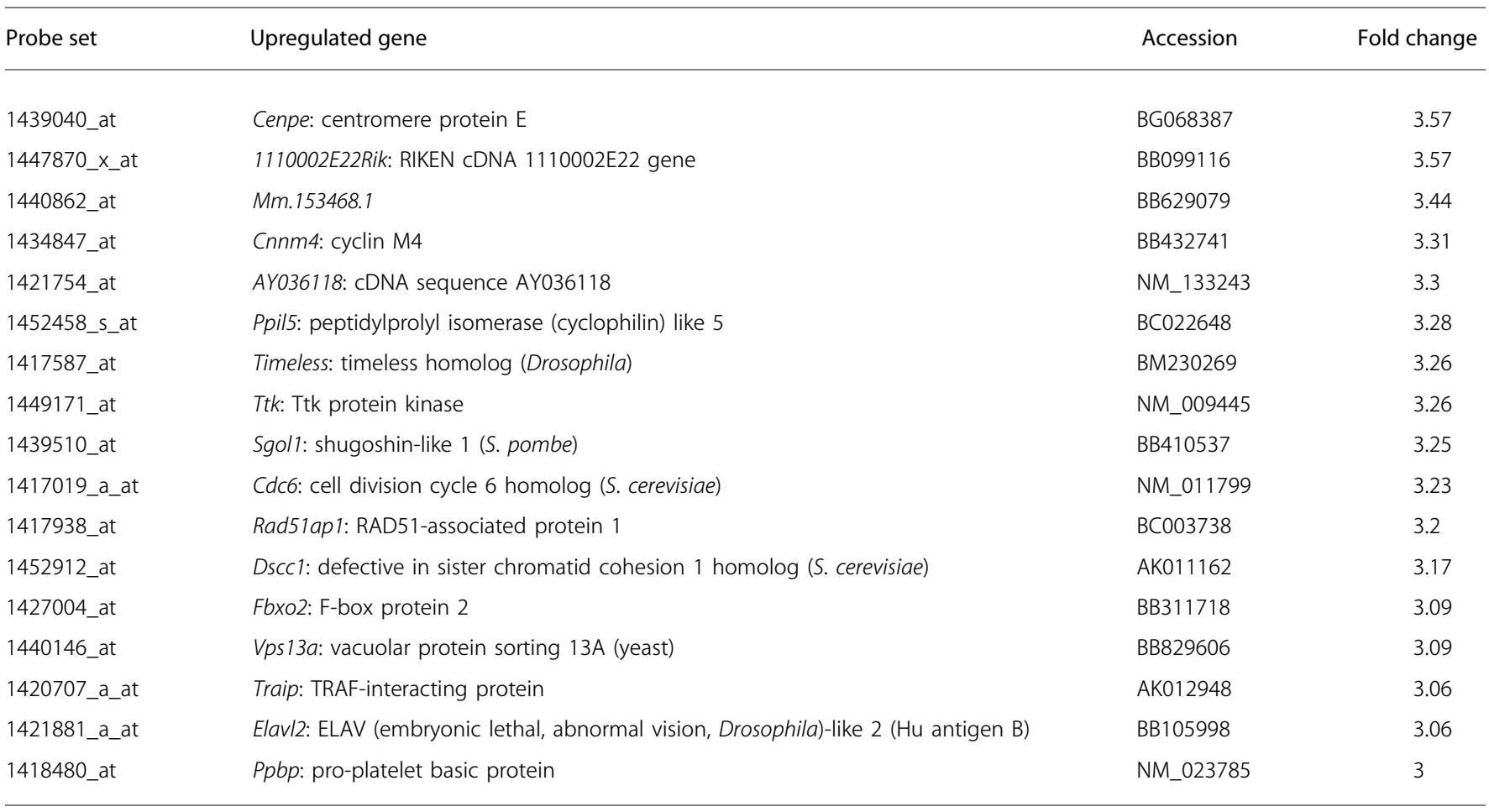

A cut off of threefold changes was used to filter the data (see Materials and Methods). Genes in bold were used for further studies, see Table 3 and Figures $1,2$.

Table 3 Genes with altered expression both in $1 \mathrm{~W}$ and $2 \mathrm{~W}$ array data sets, compared with controls

\begin{tabular}{lllrrrrrrr}
\hline Probe set & Gene & Accession & Fold change & NG & NG & 1W & 1W & 2W & 2W \\
\hline 1419063_at & Ugt8a: UDP galactosyltransferase 8A & NM_011674 & $\mathbf{5 . 2 6}$ & $\mathbf{3 5 3 . 8}$ & $\mathbf{1 6 3 . 9}$ & $\mathbf{8 8 1 . 9}$ & $\mathbf{7 1 5 . 9}$ & $\mathbf{1 3 2 7}$ & $\mathbf{1 2 5 1 . 9}$ \\
1421262_at & Lipg: lipase, endothelial & BC020991 & $\mathbf{3 . 9 5}$ & $\mathbf{5 8 4 . 9}$ & $\mathbf{7 4 8 . 7}$ & $\mathbf{2 3 3 8 . 1}$ & $\mathbf{1 8 7 2 . 4}$ & $\mathbf{2 2 8 5 . 2}$ & $\mathbf{2 8 8 2 . 8}$ \\
1427747_a_at & Lcn2: lipocalin 2 & X14607 & $-\mathbf{7 . 0 3}$ & $\mathbf{5 5 7 2 . 4}$ & $\mathbf{5 4 4 2 . 6}$ & $\mathbf{1 3 3 5 . 5}$ & $\mathbf{1 5 4 6 . 3}$ & $\mathbf{9 5 8 . 8}$ & $\mathbf{5 6 3 . 5}$ \\
1430786_at & 1110002E22Rik: RIKEN cDNA 1110002E22 gene & BE991102 & 4.39 & 531.2 & 467.5 & 2795.8 & 2011.6 & 1458.4 & 2842.1 \\
1431315_at & Hyls1: hydrolethalus syndrome 1 & BM570636 & 4.59 & 212.3 & 85.9 & 691.4 & 386.7 & 642.2 & 581.3 \\
1444199_at & Mm.45087.1 & AW046689 & 4.89 & 634.9 & 251 & 641.3 & 2593.8 & 555.9 & 3695.5 \\
1447870_x_at & 1110002E22Rik: RIKEN cDNA 1110002E22 gene & BB099116 & 3.57 & 1318.4 & 1917 & 6276.8 & 5591.4 & 3453 & 8163 \\
1448595_a_at & Bex1: brain-expressed gene 1 & NM_009052 & -3.6 & 8614.8 & 18678.4 & 3023.9 & 1035 & 6905.6 & 569.2 \\
1449751_at & Slc6a6: Solute carrier family 6 (neurotransmitter & AA589629 & 4.57 & 679.1 & 271.1 & 548 & 3369.7 & 820.2 & 3314.8 \\
& transporter, taurine), member 6 & & & & & & & \\
1455930_at & Mm.28870.2 & Bl651113 & 13.95 & 3317.7 & 2655.5 & 4788.1 & 49494.4 & 5569.2 & 89776
\end{tabular}

Columns on the right represent the raw signal intensity data for the probe sets for the indicated samples. The genes highlighted in bold were prioritized for validation on the basis of consistency of replicate data across the arrays and available annotation.

lipocalin 2 (Lcn2). There were 19 transcripts that were upregulated at 1 week including interleukin 1 receptor-like 1 (Il1rl1), EL (Lipg) and UDP galactosyltransferase 8A (Ugt8). In all, 25 genes were downregulated in week 2, including Lcn2, MMP2 and Cyclin 1 (Ccni). A total of 32 genes were upregulated at 2 weeks in HG-treated podocytes, including Ugt8 and EL (for detailed list and fold changes, see Table 1).
We next focused on identifying genes that were represented by probe sets showing consistent changes in response to HG at both 1 week (arbitrary representation of sub-acute HG stress) and 2 weeks (arbitrary representation of sustained HG stress) in our model. We were interested in this for two reasons. First, these could represent potential biomarkers that remain altered in diabetic glomerular disease. Second, these 


\section{a Downregulated}

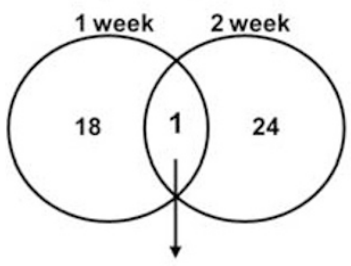

Lipocalin 2 (LCN2 or NGAL) b Upregulated

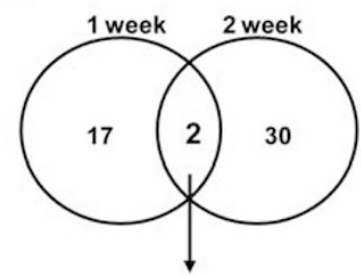

Endothelial Lipase (EL) UDP-gluronosyltransferase 8 (UGT8)

Figure 1 Common genes (a) downregulated and (b) upregulated in podocytes cultured in HG for 1 and 2 weeks compared with NG controls. Among the differentially expressed genes, only three were consistently altered at both time points. Refer to Table 1 for a detailed list of genes at each time point.

could provide insights into possible mechanisms underlying HG-mediated podocyte dysfunction. Therefore, we generated a gene list representing intersection of differentially expressed genes at both 1 and 2 weeks of HG compared with NG exposure (Table 3 ). Of the 10 genes that were differentially expressed in both the 1 week and 2 week time points compared with the NG control, only 3 genes showed consistent changes in probe sets across replicates when the raw signal intensity data were examined, and that had defined annotation information. These include neutrophil gelatinase-associated lipocalin ( $L c n 2$ also known as Ngal1) expression of which was decreased in response to HG, and EL and Ugt8, expression levels of which increased in response to HG (Figure 1).

To further understand the temporal dynamics of these alterations, and independently validate microarray results, we isolated RNA from podocytes exposed to HG at different time intervals $(6,12,18,24$ and $72 \mathrm{~h}$, and 1 and 2 weeks) and performed RT-PCR for Lcn2, EL and Ugt8 (Figure 2). Consistent with our microarray data, we found that all three genes were modulated by HG. Importantly, Lcn2 levels decreased as early as 6 hours after HG exposure and were significantly downregulated by HG at 18-hours. By 72 hours, Lcn2 was barely detectable and remained low for the remaining time points of this experiment and up to 1 and 2 weeks (Figure 2a). EL was upregulated as early as 6 hours by HG and increased by 3 - and 10 -fold at 1 week and 2 weeks, respectively, compared with control (Figure 2b). Ugt8 was upregulated by HG as early as 12 hours and remained elevated at 2 weeks of HG exposure (Figure 2c). These results validate the microarray findings and demonstrate that perturbation in expression of these genes after high-glucose exposure was a relatively early event $(<18$ hours after HG exposure) and the expression remained downregulated (for Lcn2) or upregulated (for EL and Ugt8) at longer times of HG exposure.

We next determined whether the HG responsive genes found from experiments in vitro were also altered in patients

a

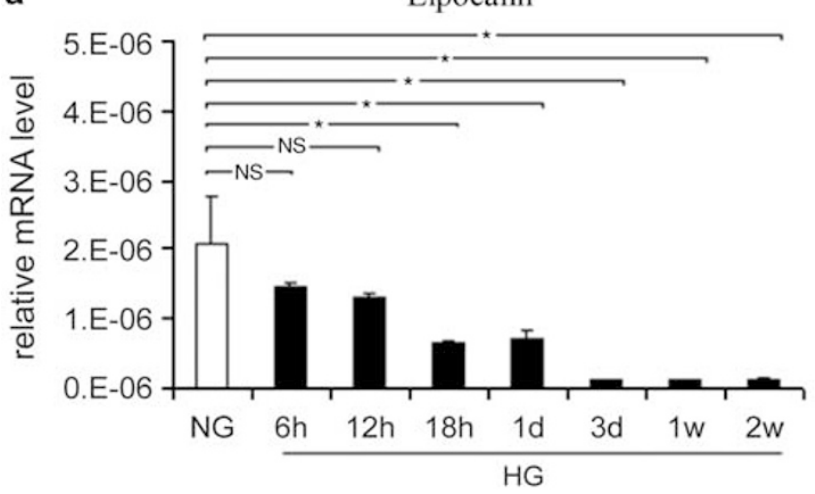

b

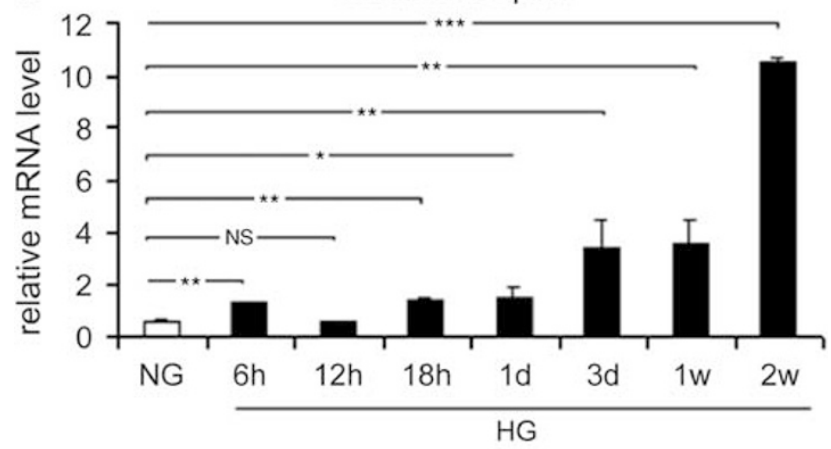

C

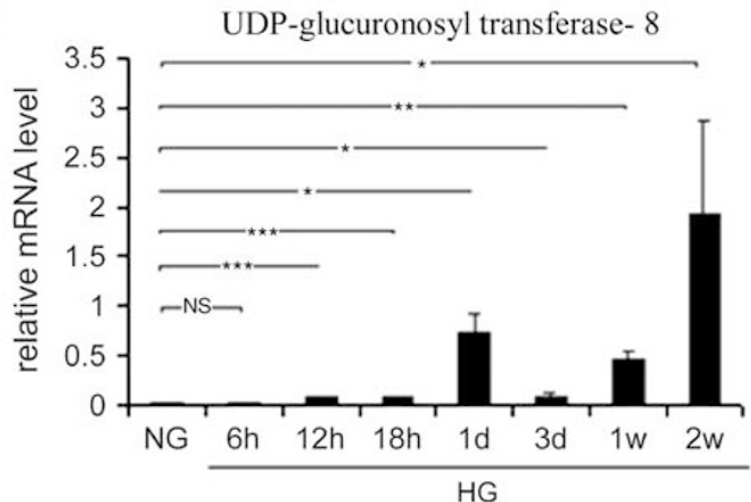

Figure 2 Effect of HG on lipocalin (a), endothelial lipase (b) and UDPglucuronosyl transferase-8 (c) mRNA expression in podocytes. Cells were incubated in NG RPMI 16 with/without 25 mM D-glucose for 6, 12, 18, 24, 48 and 72 hours, 1 week and 2 weeks. Cells were collected and assayed for target gene mRNA levels by real time-PCR. The relative mRNA expression of the target genes were normalized to $\beta$-actin control. Each point represents the mean \pm s.d. of three independent experiments performed in triplicate. ${ }^{*} P \leq 0.05 ;{ }^{* *} P \leq 0.01 ;{ }^{* * *} P \leq 0.001 ; N S$, not significant.

with diabetic nephropathy. To obtain a clearer idea at a cell-specific level, we resorted to immunohistochemistry in kidneys from diabetic or non-diabetic patients (eight diabetic and seven non-diabetic controls). Among the three differentially expressed genes, we focused on EL2 as Ugt8 antibodies were not available and Lcn2 was barely detectable in podocytes of non-diabetic patients (low abundance expression), thus rendering it unsuitable for detecting any further downregulation in situ in diabetic glomeruli. 
Consistent with our cell-culture results, we observed markedly higher EL2 expression in podocytes of almost all diabetic nephropathy patients (seven out of eight) compared with those from non-diabetics (normal; Figure 3). Immunostaining with WT1 and EL antibodies using immunoperoxidase or immunofluorescence methods confirms high expression of EL in podocytes of diabetic glomeruli. Parietal cells of diabetic glomeruli also exhibit high EL immunopositivity compared with controls and non-diabetic kidneys, but the significance of this is unclear.

We further examined whether these findings can have potential utility in DN diagnosis and performed EL immunoblotting in urine samples from diabetic nephropathy and non-diabetic control patients. EL was readily detected in urine samples of a subset of DN patients (2/4), whereas none of the control patients (3/3) show EL expression (Figure 4).

\section{DISCUSSION}

We have employed microarray analysis to delineate potential molecular pathways underlying podocyte injury in diabetic glomerulopathy using an in vitro model and confirmed a subset of the observed in vitro changes in independent timecourse experiments and in specimens from humans with diabetic nephropathy. Out of a total of 95 transcripts that were differentially expressed upon HG exposure of 1 or 2 weeks, we found that three remained altered at both 1 and 2 weeks (Lcn2, Ugt8 and EL), suggesting that these are promising candidates as biomarkers or potential contributors to the pathogenesis of diabetic glomerular disease. Time-course RT-PCR experiments revealed that changes in mRNAs of these genes occur shortly after exposure to HG and remain altered, which may have implications in early and late detection and pathogenesis.

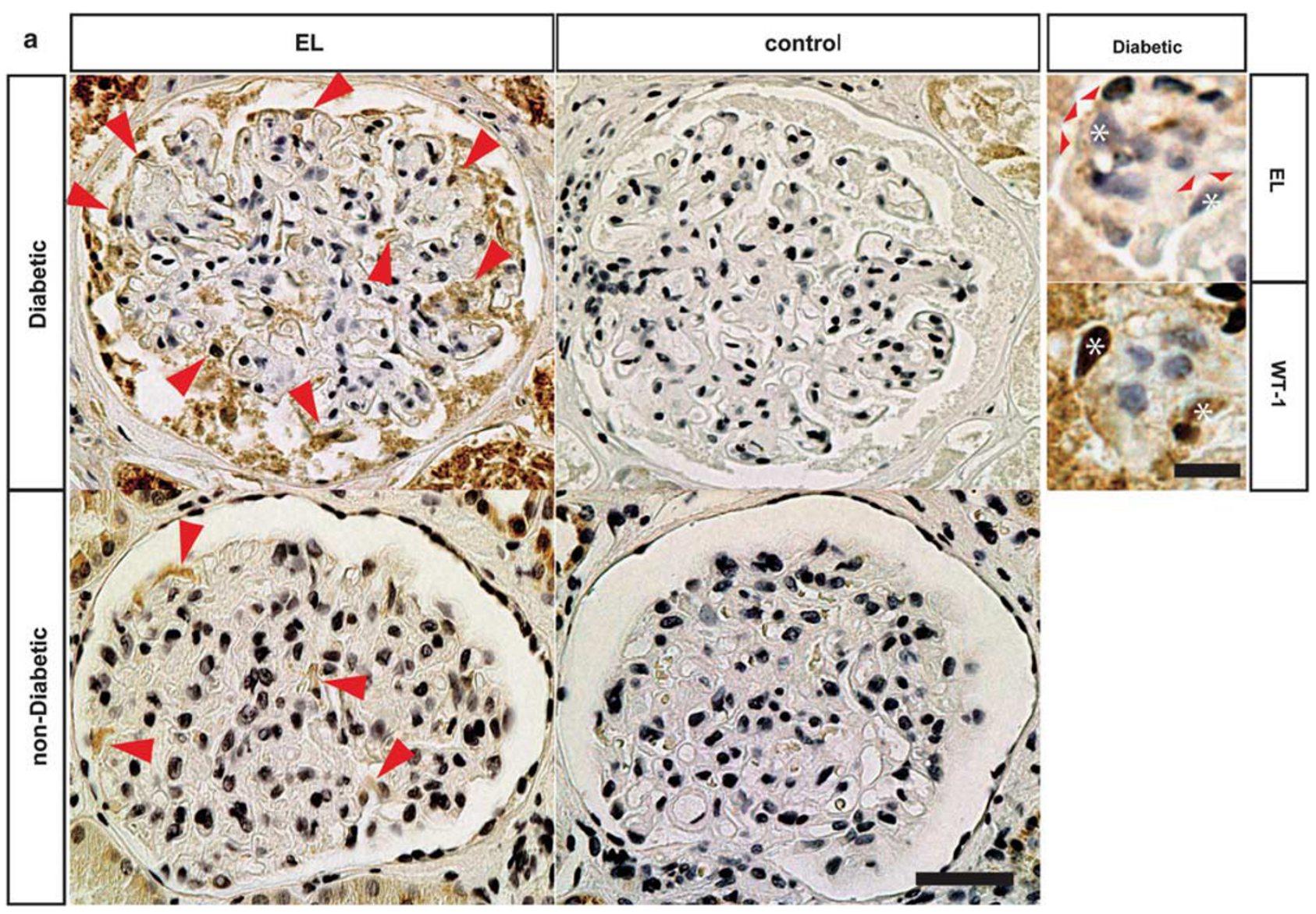

Figure 3 High endothelial lipase expression in kidney sections from diabetic patients compared with non-diabetic kidneys. EL immunostaining was performed on formalin-fixed paraffin embedded (a) or cryopreserved (b) biopsies from patients with diabetic nephropathy (eight) or non-diabetics (seven) (see Materials and Methods). The primary antibody was omitted in the control panel. All diabetic patients except one had increased EL immunopositivity in podocytes. Representative images from three diabetic and two non-diabetic patients are shown. (a) Immunoperoxidase staining (brown) with anti-EL antibodies show increased EL expression (red arrows) in podocytes and parietal cells of diabetic compared with non-diabetic glomeruli. (Scale bar $=50 \mu \mathrm{m}$.) The high power images on the right (scale bar $=10 \mu \mathrm{m}$ ) are adjacent sections from a diabetic kidney stained with anti-EL or anti-WT-1 (as a podocyte marker) that show colocalization of EL (red arrowheads) and WT-1 (dark-brown nuclear staining) in same cells (asterisks) supporting that EL and WT-1 are expressed in the podocytes. (b) EL immunofluorescence (red) on non-diabetic and diabetic kidney tissues also shows increased EL expression in diabetic glomeruli (lower panel), compared with non-diabetic glomeruli (upper panel). Control shows no glomerular staining. WT1 (green nuclear, arrowheads) immunostaining confirms that EL-expressing cells (arrows, cytoplasm) are podocytes. (Scale bar $=50 \mu \mathrm{m}$.) The tubulointerstitial staining is non-specific, as it is present in controls in both $\mathbf{a}$ and $\mathbf{b}$. 

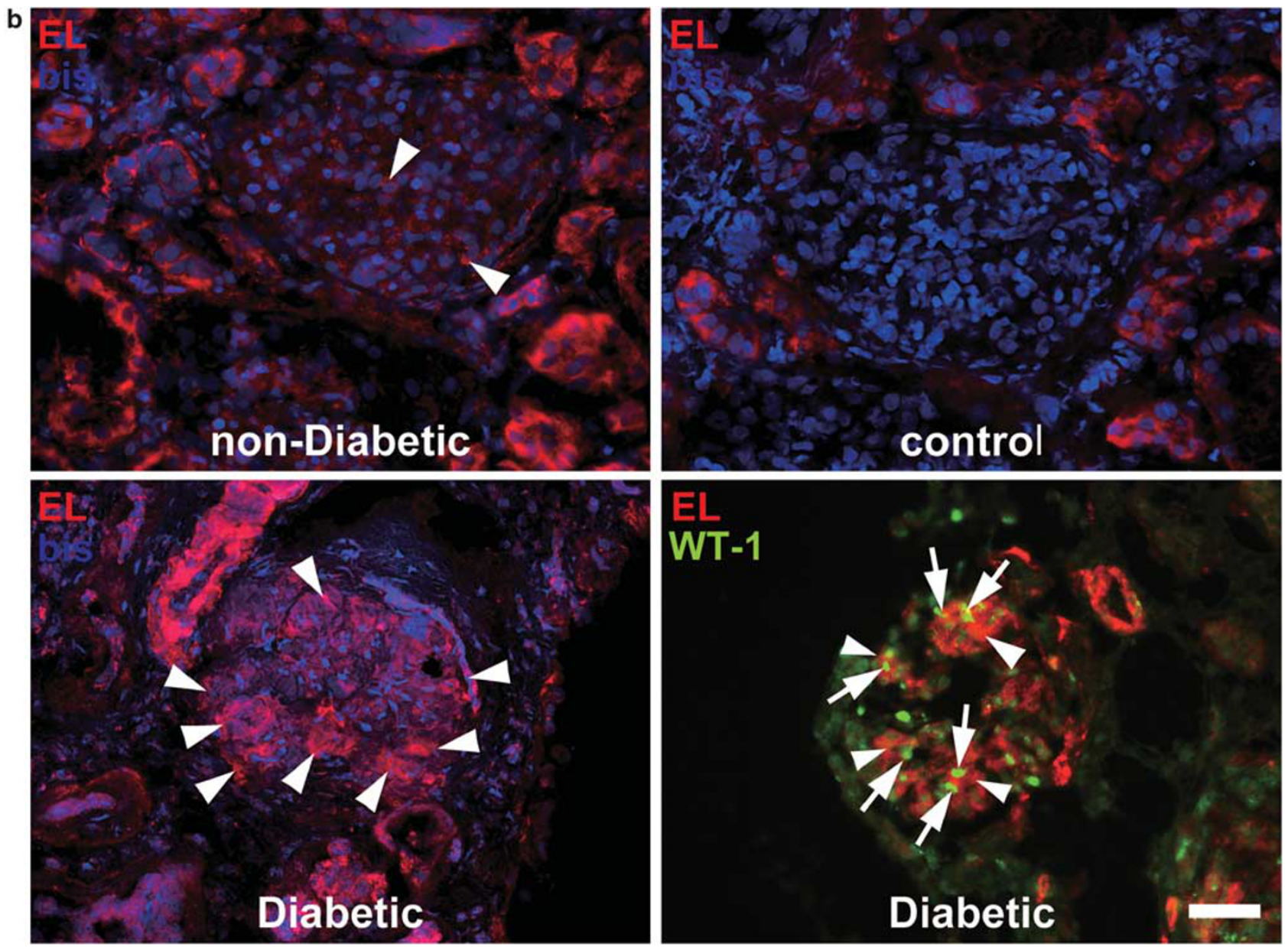

Figure 3 Continued.

We discovered that Lcn2 expression exhibited a timedependent decrease in podocytes, beginning as early as 6 hours after HG treatment. LCN2 is a member of the lipocalin superfamily that forms a complex with iron-binding siderophores. This complex has a role during nephrogenesis by promoting the conversion of renal progenitors into tubules. ${ }^{31}$ Further, it is also highly upregulated in tubular cells after acute kidney injury. ${ }^{32}$ These results support the idea that upregulation of Lcn2 is important for survival or regeneration after injury from stress. This idea is further supported by the observation that recombinant $L \mathrm{cn} 2$ reduces proximal tubular injury in an ischemia-reperfusion injury model by inhibiting apoptosis. ${ }^{33}$ The decreased expression of Lcn2 observed in our study was unexpected considering its upregulation in other injury models; however, our independent validation using quantitative RT-PCR at several time points supported the observed Lcn2 downregulation upon HG exposure . This observation suggests that failure of podocytes to sustain normal or high levels of Lcn2 after HG exposure may be a potential mechanism of podocyte injury and apoptosis induced by HG. LCN2 also binds to MMP-9 and protects this extracellular matrix remodeling enzyme from autodegradation. ${ }^{34}$ Whether reduced expression of
LCN2 contributes to GBM thickening and extracellular matrix accumulation (hallmark lesions of diabetic glomerulosclerosis) by increasing autodegradation of MMP-9 or other metalloproteinases remains to be seen.

Our discovery of upregulation of EL in vitro and in human samples has important clinical implications. EL, the most recently discovered member of the lipase gene family, is an important negative modulator of high-density lipoprotein (HDL). EL is also involved in inflammatory state by promoting monocyte adhesion to the vascular endothelium. ${ }^{35,36}$ Recent data indicate that HDL inhibits apoptosis, lipid oxidation, cytokine and adhesion molecule production. ${ }^{37}$ As in vitro and in vivo studies have linked HDL to both diabetes mellitus and inflammation, it is possible that increased EL in our study of podocyte HG exposure may be a mechanism of HDL-mediated or cytokine-mediated effects in diabetic glomerulopathy. Further, HDL is thought of as protective against formation of extrarenal vascular calcifications, a common and serious complication in patients with chronic kidney disease. ${ }^{38}$ Therefore, it is possible that elevated EL in diabetic glomerulopathy mediates low HDL-associated increased vascular calcifications. Importantly, we demonstrate for the first time that podocytes may be a significant 
a

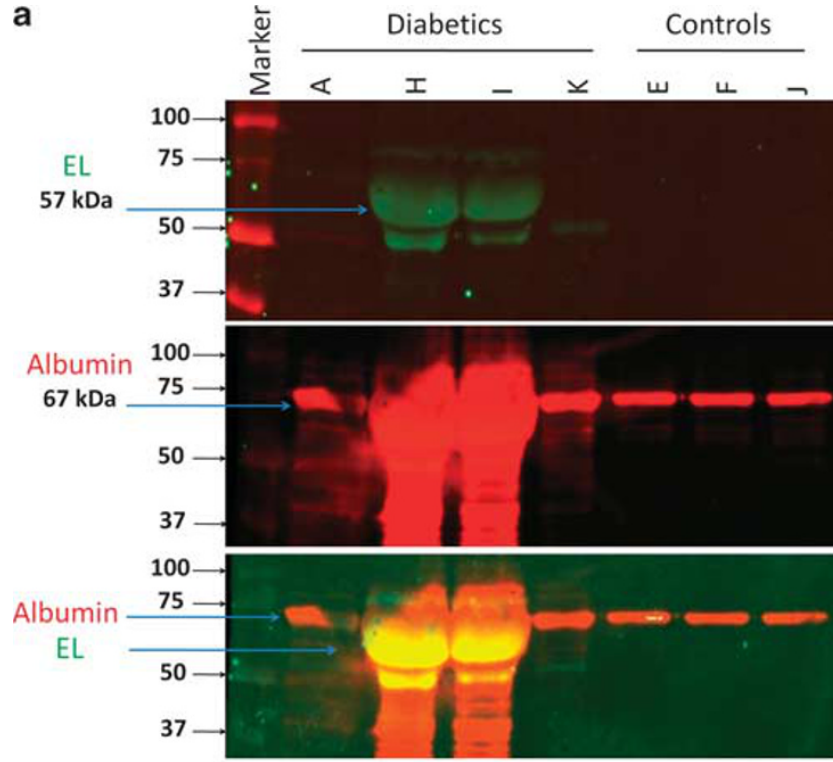

b

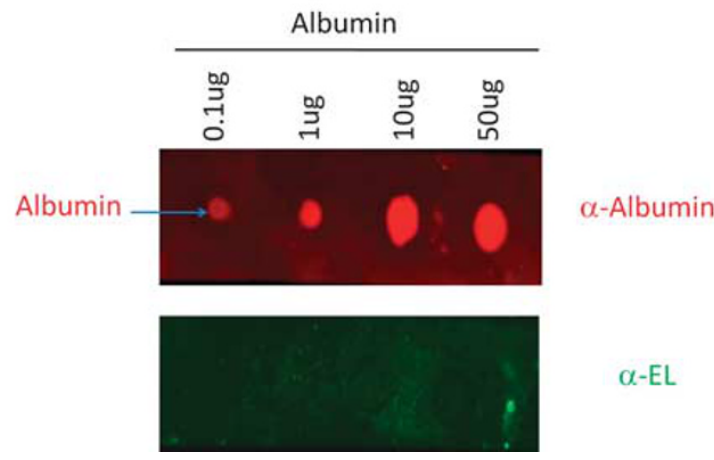

Figure $4 \mathrm{EL}$ is readily detected in urine of diabetic nephropathy patients. (a) Immunoblot analysis for EL ( $57 \mathrm{kDa})$ and albumin $(67 \mathrm{kDa})$ in urine samples of diabetic (lanes A, H, I and K) and non-diabetic controls (lanes E, F and J). High levels of EL (green) are detected in urine samples of two diabetic patients ( $\mathrm{H}$ and I). Controls show no EL excretion. Albumin (red) was detected in all samples. The lower panel shows distinct migration of EL and albumin. (b) EL antibody does not crossreact with albumin. Dot blot pattern of EL and albumin immunostaining to different amounts of purified human albumin protein shows no immunostaining with EL antibody further confirming that EL detection is specific.

source of EL, a protein normally secreted by vascular endothelial and smooth cells or macrophages (cell types typically not present in the glomerulus), after HG exposure. The fact that EL can be readily detected in human urine samples in a subset of diabetic patients also supports its potential use in diabetic nephropathy diagnosis. Further large-scale studies are warranted to determine the diagnostic utility of aberrant EL expression in diabetic and non-diabetic nephropathy at different stages of the disease.

Although LCN2 and EL can be potentially linked to HGmediated changes related to diabetic nephropathy, currently no such information is available for UGT8. UGT8 consists of a super family of enzymes that catalyze glucuronidation. ${ }^{39}$ In particular, UGT8 family catalyzes the transfer of galactose to ceramide, a key enzymatic step in the the biosynthesis of galactocerebrosides. Galactocerebrosides are abundant sphingolipids of the myelin membrane of the central and peripheral nervous system, ${ }^{40}$ and Ugt8 is also present in the kidney during metanephric development. ${ }^{41}$ Ugt8 deficiency results in a spectrum of neurological symptoms characterized by tremors, ataxia, progressive hindlimb paralysis and vacuole formation in ventral spinal cord. ${ }^{42}$ Increased ceramide levels have also been shown to increase apoptosis in a number of systems. ${ }^{43}$ On the other hand, Ugt8 upregulation is associated with increased metastatic potential to lung by specifically enhancing the ability to metastasize to, colonize and survive within the lung. ${ }^{44}$ It should be noted that podocytes have been touted as neuronal counterparts in the kidney, and the increased Ugt8 levels may be protective compensatory response to prevent podocyte degeneration by enhancing their survival, perhaps similar to its role in the nervous system by regulating lipid metabolism. Future studies would be needed to explore a potential relationship of Ugt8 to diabetic glomerulopathy or podocyte function.

In conclusion, our study identified podocyte specific genes that are regulated by acute and sustained HG exposure. Although some of these have been previously associated with diabetic glomerulopathy thus validating our strategy, we found several novel ones. We confirmed a subset of these that may cause deranged lipid metabolism and impaired injury response in podocytes and contribute to diabetic nephropathy. Further, we show data for potential application of these in diagnosis of diabetic nephropathy in human specimens. Thus, these studies provide new molecular insights into the role of podocytes in diabetic renal disease and support the notion that diabetes with regard to the glomerulus is another podocytopathy. ${ }^{45}$

\section{ACKNOWLEDGEMENTS}

We thank Amanda Knoten, Angela Lluka and Adee Heiman for excellent technical assistance, and Mary Hoffman, Jessica Vanwinkle and Daniel Martin for human specimen processing and acquisition. We thank Dr George Jarad for human purified albumin and albumin antibodies. We are grateful to Mark Watson and Laboratory of Translational Pathology at Washington University School of Medicine for providing core services for microarray and laser capture microscopy studies, and to Dr Erwin P Bottinger for providing laboratory resources to culture podocytes for RT-PCR experiments. This work was partly supported by Core services provided by an NIH George M. O'Brien Center for Kidney Disease Research grant (P30-DK079333) to Washington University, NIH grant DK081644 (to $\mathrm{S}$ Jain) and interdepartmental research funds to $\mathrm{H}$ Liapis. Part of this work was presented in an abstract form at the annual United States and Canadian Academy of Pathology (USCAP) 2006 and International Podocyte Conference (2006).

\section{DISCLOSURE/CONFLICT OF INTEREST}

The authors declare no conflict of interest.

1. Wolf G, Chen S, Ziyadeh FN. From the periphery of the glomerular capillary wall toward the center of disease: podocyte injury comes of age in diabetic nephropathy. Diabetes 2005;54:1626-1634. 
2. Reddy GR, Kotlyarevska K, Ransom RF, et al. The podocyte and diabetes mellitus: is the podocyte the key to the origins of diabetic nephropathy? Curr Opin Nephrol Hypertens 2008;17:32-36.

3. Marshall MS. The podocyte: a major player in the development of diabetic nephropathy? Horm Metab Res 2005;37(Suppl 1):9-16.

4. Susztak K, Raff AC, Schiffer $M$, et al. Glucose-induced reactive oxygen species cause apoptosis of podocytes and podocyte depletion at the onset of diabetic nephropathy. Diabetes 2006;55:225-233.

5. Stitt-Cavanagh E, MacLeod L, Kennedy C. The podocyte in diabetic kidney disease. ScientificWorldJournal 2009;9:1127-1139.

6. Ziyadeh FN. Different roles for TGF-beta and VEGF in the pathogenesis of the cardinal features of diabetic nephropathy. Diabetes Res Clin Pract 2008;82(Suppl 1):S38-S41.

7. Schiffer $M$, Mundel $P$, Shaw AS, et al. A novel role for the adaptor molecule CD2-associated protein in transforming growth factor-betainduced apoptosis. J Biol Chem 2004;279:37004-37012.

8. Li Y, Kang YS, Dai C, et al. Epithelial-to-mesenchymal transition is a potential pathway leading to podocyte dysfunction and proteinuria. Am J Pathol 2008;172:299-308.

9. Marrero B, Banes-Berce AK, Stern DM. Role of the JAK/STAT signaling pathway in diabetic nephropathy. Am J Physiol Renal Physiol 2006; 290:F762-F768.

10. Noh $\mathrm{H}$, King $\mathrm{GL}$. The role of protein kinase $\mathrm{C}$ activation in diabetic nephropathy. Kidney Int Suppl 2007;106:S49-S53.

11. Forbes JM, Fukami K, Cooper ME. Diabetic nephropathy: where hemodynamics meets metabolism. Exp Clin Endocrinol Diabetes 2007 115:69-84.

12. Schleicher ED, Weigert C. Role of the hexosamine biosynthetic pathway in diabetic nephropathy. Kidney Int Suppl 2000;77:S13-S18.

13. Daniels MC, McClain DA, Crook ED. Transcriptional regulation of transforming growth factor beta1 by glucose: investigation into the role of the hexosamine biosynthesis pathway. Am J Med Sci 2000;319:138-142.

14. Goldberg $\mathrm{HJ}$, Whiteside $\mathrm{Cl}$, Fantus IG. The hexosamine pathway regulates the plasminogen activator inhibitor-1 gene promoter and Sp1 transcriptional activation through protein kinase $C \beta 1$ and $\delta$. J Biol Chem 2002;277:33833-33841.

15. Müller-Deile J, Worthmann K, Saleem M, et al. The balance of autocrine VEGF-A and VEGF-C determines podocyte survival. Am J Physiol Renal Physiol 2009;297:F1656-F1667.

16. Satchell SC, Harper SJ, Tooke JE, et al. Human podocytes express angiopoietin 1, a potential regulator of glomerular vascular endothelial growth factor. J Am Soc Nephrol 2002;13:544-550.

17. Li JM, Shah AM. ROS generation by nonphagocytic NADPH oxidase: potential relevance in diabetic nephropathy. J Am Soc Nephrol 2003;14(8 Suppl 3):S221-S226.

18. Nyengaard JR, Ido $\mathrm{Y}$, Kilo $\mathrm{C}$, et al. Interactions between hyperglycemia and hypoxia: implications for diabetic retinopathy. Diabetes 2004;53: 2931-2938.

19. De Petris L, Hruska KA, Chiechio S, et al. Bone morphogenetic protein-7 delays podocyte injury due to high glucose. Nephrol Dial Transplant 2007;22:3442-3450.

20. Fan $Q$, Shike $T$, Shigehara $T$, et al. Gene expression profiles in diabetic KK/Ta mice. Kidney Int 2003;64:1978-1985.

21. Wilson $\mathrm{KH}$, Eckenrode $\mathrm{SE}, \mathrm{Li} \mathrm{QZ}$, et al. Microarray analysis of gene expression in the kidneys of new- and post-onset diabetic NOD mice. Diabetes 2003;52:2151-2159.

22. Susztak K, Bottinger E, Novetsky A, et al. Molecular profiling of diabetic mouse kidney reveals novel genes linked to glomerular disease. Diabetes 2004;53:784-794.

23. Baelde HJ, Eikmans M, Doran PP, et al. Gene expression profiling in glomeruli from human kidneys with diabetic nephropathy. Am Kidney Dis 2004;43:636-650.
24. Morrison J, Knoll $\mathrm{K}$, Martin $\mathrm{JH}$, et al. Effect of high glucose on gene expression in mesangial cells: upregulation of the thiol pathway is an adaptational response. Physiol Genomics 2004;17:271-282.

25. Han SH, Yang S, Jung DS, et al. Gene expression patterns in glucose-stimulated podocytes. Biochem Biophys Res Commun 2008;370:514-518.

26. Mundel P, Reiser J, Zúñiga Mejía Borja A, et al. Rearrangements of the cytoskeleton and cell contacts induce process formation during differentiation of conditionally immortalized mouse podocyte cell lines. Exp Cell Res 1997;236:248-258.

27. Jain S, Suarez AA, McGuire J, et al. Expression profiles of renal dysplasia reveal new insights in renal development and disease. Pediatr nephrol 2007;22:962-974.

28. Jains A, Dubes R. Algorithms for Clustering Data. Prentice-Hall: Englewood Cliffs NJ, 1988.

29. Li C, Wong WH. Model-based analysis of oligonucleotide arrays: expression index computation and outlier detection. PNAS 2001;98: 31-36.

30. Livak KJ, Schmittgen TD. Analysis of relative gene expression data using real-time quantitative PCR and the $2^{-\Delta \Delta C t}$ method. Methods 2001;25:402-408.

31. Challen GA, Martinez G, Davis MJ, et al. Identifying the molecula phenotype of renal progenitor cells. J Am Soc Nephrol 2004;5: 2344-2357.

32. Mishra J, Ma Q, Prada A, et al. Identification of neutrophil gelatinaseassociated lipocalin as a novel early urinary biomarker for ischemic renal injury. J Am Soc Nephrol 2003;14:2534-2543.

33. Yan L, Borregaard N, Kjeldsen L, et al. The high molecular weight urinary matrix metalloproteinase activity is a complex of gelatinase B/ MMP-9 and neutrophil gelatinase-associated lipocalin (NGAL). J Biol Chem 2005;276:37258-37265.

34. Paradis $M E$, Badellino $\mathrm{KO}$. Endothelial lipase is associated with inflammation in humans. J Lipid Res 2006;47:2808-2813.

35. Broedl UC, Maugeais C. Effects of nonlipolytic ligand function of endothelial lipase on high density lipoprotein metabolism in vivo. J Biol Chem 2003;278:40688-40693.

36. Ruge T, Neuger L. Lipoprotein lipase in the kidney: activity varies widely among animal species. Am J Physiol Renal Physiol 2004;287: F1131-F1139.

37. Rohrer L, Hersberger M, Von Eckardstein A. High density lipoproteins in the intersection of diabetes mellitus, inflammation and cardiovascular disease. Curr Opin Lipidol 2004;15:269-278.

38. Torres PA. Origin of the mediacalcosis in kidney failure. J Mal Vasc 2009;34:204-210.

39. Yoshikazu E, Koichi U, Ohnishi A, et al. Transcriptional enhancement of udp-glucuronosyltransferase form 1 A2 (UGT1A2) by nuclear factor I-A (NFI-A) in rat hepatocytes. J Biochem 2005;138:313-325.

40. Yurkova I, Kisel M. Free-radical fragmentation of galactocerebrosides: a MALDI-TOF mass spectrometry study. Chem Phys Lipids 2005;134:41-49.

41. Challen G, Gardiner B, Caruana G, et al. Temporal and spatia transcriptional programs in murine kidney development. Physiol Genomics 2005;23:159-171.

42. Guttmacher AE, Collins FS. Genomic medicine: a primer. N Engl J Med 2002;347:1512-1520.

43. WU BX, Clarke CJ, Hannun YA. Mammalian neutral sphingomyelinases: regulation and roles in cell signaling responses. Neuromolecular Med 2010; Jun 16 (e-pub ahead of print).

44. Landemaine $T$, Jackson A, Bellahcène $A$, et al. A six-gene signature predicting breast cancer lung metastasis. Cancer Res 2008;68:6092-6099.

45. Barisoni L, Schnaper HW, Kopp JB. Advances in the biology and genetics of the podocytopathies: implications for diagnosis and therapy. Arch Pathol Lab Med 2009;133:201-216. 